\title{
Stochastic description of the dynamics of a random-exchange Heisenberg chain
}

\author{
M. H. Vainstein, R. Morgado, and F. A. Oliveira \\ Instituto de Física and Núcleo de Supercomputação e Sistemas Complexos, \\ ICCMP, Universidade de Brasília, CP 04513, 70919-970, Brasília-DF, Brazil \\ F.A.B.F. de Moura * and M. D. Coutinho-Filho \\ Laboratório de Física Teórica e Computacional, Departamento de Física, \\ Universidade Federal de Pernambuco, 50670-901 Recife, PE, Brazil and \\ (*)Departamento de Física, Universidade Federal de Alagoas, 57072-970 Maceió, AL, Brazi *
}

\begin{abstract}
We study the diffusion process in a Heisenberg chain with correlated spatial disorder, with a power spectrum in the momentum space behaving as $k^{-\beta}$, using a stochastic description. It establishes a direct connection between the fluctuation in the spin-wave density of states and the noise density of states. For continuous ranges of the exponent $\beta$, we find super-diffusive and ballistic spinwave motions. Both diffusion exponents predicted by the stochastic procedure agree with the ones calculated using the Hamiltonian dynamics.
\end{abstract}

\section{INTRODUCTION}

In the last decades, a considerable number of dynamical systems has been studied and a great deal of attention has been paid to the analysis of its transport properties. In particular, the study of diffusion and transport properties of physical systems with short or long-range correlations in the disorder distribution has attracted a renewed interest 1 , $2,3,4,5,6,67,8,9,10,11,12$. For instance, the unexpected high conductance of several doped quasi-one-dimensional polymers was explained by assuming pairwise correlations in the disorder distribution [3]. Similarly, the suppression of Anderson localization was recently confirmed experimentally in semiconductor superlattices with intentional correlated disorder [13]. Further, it was demonstrated that long-range correlations in site also act towards the delocalization of 1D quasiparticle states 10, 11]. The 1D Anderson model with long-range correlated diagonal disorder displays a finite phase of extended states in the middle of the band of allowed energies, with two mobility edges separating localized and extended states [10]. This result was experimentally validated by microwave transmission spectra through a single-mode waveguide with inserted correlated scatterers [14]. The above results have motivated the study of other model systems that can be mapped onto the Anderson model, such as magnetic [15] and harmonic chains [16].

In the context of stochastic process, Morgado et al. 1], studied diffusion in systems with long-range time correlation. First, they establish a direct connection between the noise density of states, $\rho_{n}(\omega)$, and the diffusive process. Second, they conjecture that the dynamics of a Hamiltonian system with space correlated disorder could be described by the same formalism if one supposes that the fluctuation in the density of states of the quasi-particle or elementary excitation, $\rho_{F}(\omega)$, plays the same role as $\rho_{n}$ in the stochastic description. In this work, we present a numerical analysis of the validity of this conjecture. We study it for the one-dimensional quantum Heisenberg model exhibiting long-range correlations in the random exchange couplings. For continuous ranges of the degree of correlation, this system presents super-diffusive and ballistic motions [15]. Here, we provide numerical evidence that the Hamiltonian description and the stochastic one can be unified through the referred conjecture, thus confirming early expectations [1].

\section{STOCHASTIC AND HAMILTONIAN DESCRIPTIONS}

Let us suppose that the equation of motion for an operator $A$ can be cast in the form [17, 18, 19, 20]

$$
\frac{d A(t)}{d t}=-\int_{0}^{t} \Gamma\left(t-t^{\prime}\right) A\left(t^{\prime}\right) d t^{\prime}+h(t)
$$

${ }^{*}$ Corresponding author:(F.A.B.F. de Moura) fidelis@df.ufal.br 
where $h(t)$ is a stochastic noise subject to the conditions $\langle h(t)\rangle=0,\langle h(t) A(0)\rangle=0$, and to the fluctuation-dissipation theorem [17]:

$$
C_{h}(t)=<h(t) h(0)>=<A^{2}>\Gamma(t) .
$$

Here, $\langle\ldots\rangle$ indicates an ensemble average in thermal equilibrium. In principle, the presence of the kernel $\Gamma(t)$ allows us to study a large number of correlated processes. For example, in analogy with the usual Langevin's equation, we can study the asymptotic behavior of the second moment of the variable,

$$
\sigma(t)=\int_{0}^{t} A(s) d s
$$

namely

$$
\lim _{t \rightarrow \infty} \frac{<\sigma^{2}(t)>}{t^{\alpha}}=K,
$$

where $K$ is a constant. In Eq. (4), we have $\alpha=1$ for normal diffusion; for sub- and super-diffusion, $\alpha<1$ and $\alpha>1$, respectively.

The generalized field, $h(t)$, in Eq. (11) can be modeled by a thermal bath composed of harmonic oscillators; consequently, according to Eq. (2), the memory function can be written as

$$
\Gamma(t)=\int \rho_{n}(\omega) \cos (\omega t) d \omega,
$$

where $\rho_{n}(\omega)$ is the noise density of states. It has been proved [1] that If the Laplace transform of the memory function of a unidimensional system behaves as

$$
\widetilde{\Gamma}(z \rightarrow 0) \propto z^{\nu}
$$

then the diffusion exponent is

$$
\alpha=\nu+1
$$

In disordered Hamiltonian systems the diffusion process can be studied through direct integration of the equations of motion [15, 16]. Now then, how can we assure that the two approaches are compatible and lead to the same results? For those systems the density of states of the quasi-particle or of the elementary excitation, $\mathbf{D}(E)$, plays the most significant role. However, it displays fluctuations, which are intrinsically connected to the diffusive behavior. Here, we conjecture that, for the relaxation processes, the fluctuation in the density of states, $\rho_{F}(E)$, plays the same role as the noise density of states in the stochastic process, thus

$$
\rho_{n}(E) \leftrightarrow \rho_{F}(E) .
$$

Consequently, if we calculate $\rho_{F}(E)$ we can obtain the memory function through Eq. (5) and the diffusion exponent using Eqs. (6) and (7). Below, we explore these ideas in the framework of the referred magnetic Hamiltonian system [15].

The one-dimensional Heisenberg chain with long-range correlated random exchange couplings can be described by the Hamiltonian [15]

$$
H=-\sum_{l=1}^{N} J_{l} \mathbf{S}_{l} \cdot \mathbf{S}_{l+1},
$$

where $S=1 / 2$, and the exchange integral is defined by

$$
J_{l}=\sum_{k=1}^{N / 2} \Delta(k)^{1 / 2} \cos \left(\pi k l / N+\phi_{k}\right) .
$$

Here, $\phi_{k}$ are random phases, and $\Delta(k)$ is a power law spectrum in the $\mathrm{k}$ space given by

$$
\Delta(k) \propto k^{-\beta} .
$$




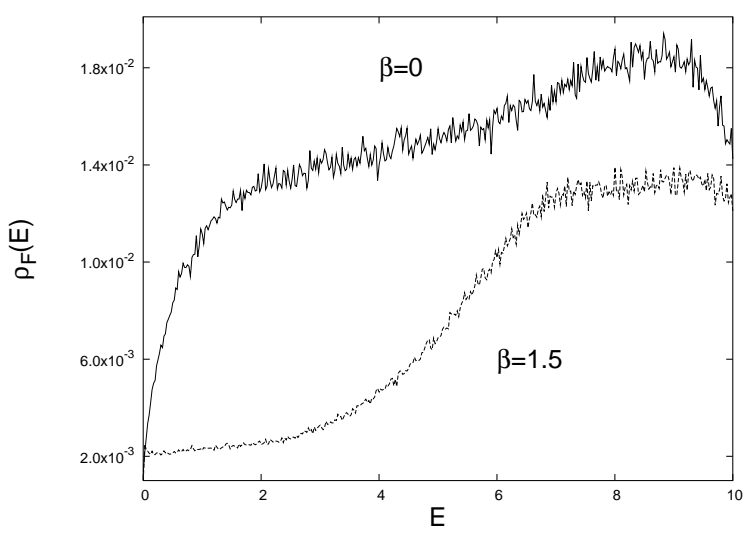

FIG. 1: Fluctuations in the density of states $\rho_{F}(E)$ as function of $E$ for $\beta=0$ and $\beta=1.5$. The average is over 900 samples. For $\beta=0$, the curve suggests a power-law for low energies, while for $\beta=1.5$, a plateau is observed.

By using a numerical renormalization group technique, it was predicted that this Hamiltonian supports a phase of low-energy extended spin waves in the strongly correlated regime $\beta>1$. Associated with these non-scattered modes, the spread of an initially localized wave packet displays a ballistic behavior in the long time limit [15]; i.e. Eq. (4) with $\alpha=2$. In the weakly correlated regime, $0<\beta<1$, a super-diffusive behavior is obtained with $\alpha=1.5$. It is worth mentioning that the wave packet dynamics was investigated by means of a direct integration of the equations of motion using a fourth order Runge-Kutta method [12, 15]. In this case the site spin operator $\mathbf{S}_{l}$ is here identified with operator $A$ in the stochastic formalism. Therefore if conjecture (8) is true the exponent of Eq. (7) should match the one calculated using the Hamiltonian dynamics [15].

\section{NUMERICAL ANALYSIS}

Now we describe the numerical method used to obtain the dynamical behavior, which consists in calculating the Laplace transform $\widetilde{\Gamma}(z)$ of the memory function $\Gamma(t)$ defined by Eq. (5). However, we first compute the function $\rho_{F}(E)$, defined by

$$
\rho_{F}(E)=<\mathbf{D}(E)^{2}>_{C}-<\mathbf{D}(E)>_{C}^{2},
$$

where $\langle\ldots\rangle_{C}$ is an average over chains with different random sets of exchange integrals. To that end, we use Dean's numerical method 21] to obtain the spin-wave density of states, $\mathbf{D}(E)$, and the corresponding $\rho_{F}(E)$, shown in Fig. 1 , for $\beta=0$ and $\beta=1.5$. It is clear from the data that, for low energies, the fluctuations $\rho_{F}(E)$ in the correlated case, $\beta=1.5$ in this figure, are smaller than the uncorrelated case $(\beta=0)$. For $\beta>1$, it was shown in ref [15] that the low energy states are extended, and that the density of states has a similar behavior as that of the pure chain. Therefore, we concluded that the finite value for $\rho_{F}(E)$ obtained in the correlated case $(\beta=1.5)$ was not stable in the thermodynamic limit. In order to obtain the most probable value of the fluctuations in the correlated case, we perform a scaling analysis of the function $\rho_{F}(E)$ for chains with distinct lengths.

Hence, we consider the following scaling function

$$
\Theta\left(E, N_{1}, N_{2}\right)=\exp \left(-\left[\rho_{F}\left(E, N_{1}\right) N_{1}-\rho_{F}\left(E, N_{2}\right) N_{2}\right]\right),
$$

with $N_{1}>N_{2}$. This method has been successfully used to obtain the behavior of a harmonic chain in the thermodynamic limit [16]. In Fig. 2] we show the scaling as a function of the energy $E$. If $\Theta\left(E, N_{1}, N_{2}\right)$ vanishes when we increase the chain size, the fluctuations are finite in the thermodynamic limit. However, if $\Theta\left(E, N_{1}, N_{2}\right) \approx 1$, the fluctuations $\rho_{F}(E)$ decrease to zero when we increase the chain size. In order to clarify this figure, we interpolated the data using a Bezier interpolation function. In the insets we show the original data without interpolations. We use $N_{2}=1.0 \cdot 10^{4}$, and three distinct values for $N_{1}$. Fig. 2(a) displays the results for $\beta=0$. From this figure we can see that $\Theta\left(E, N_{1}, N_{2}\right) \ll 1$ in all energy ranges, except at $E=0$. This indicates the existence of an extended state at this energy, which is determinant in the super-diffusive behavior. Figure 2(b) shows $\Theta\left(E, N_{1}, N_{2}\right)$ for $\beta=1.5$. In the range of low extended states, $0<E<E_{c}, \Theta\left(E, N_{1}, N_{2}\right) \rightarrow 1$, meaning that $\rho_{F}(E) \rightarrow 0$ in the thermodynamic limit. Above $E_{c}, \Theta\left(E, N_{1}, N_{2}\right) \ll 1$. The presence of a finite range of energy with extended states, $\rho_{F}(E)=0$ for $0<E<E_{c}\left(E_{c} \approx 4\right)$, is responsible for the ballistic behavior [1]. 

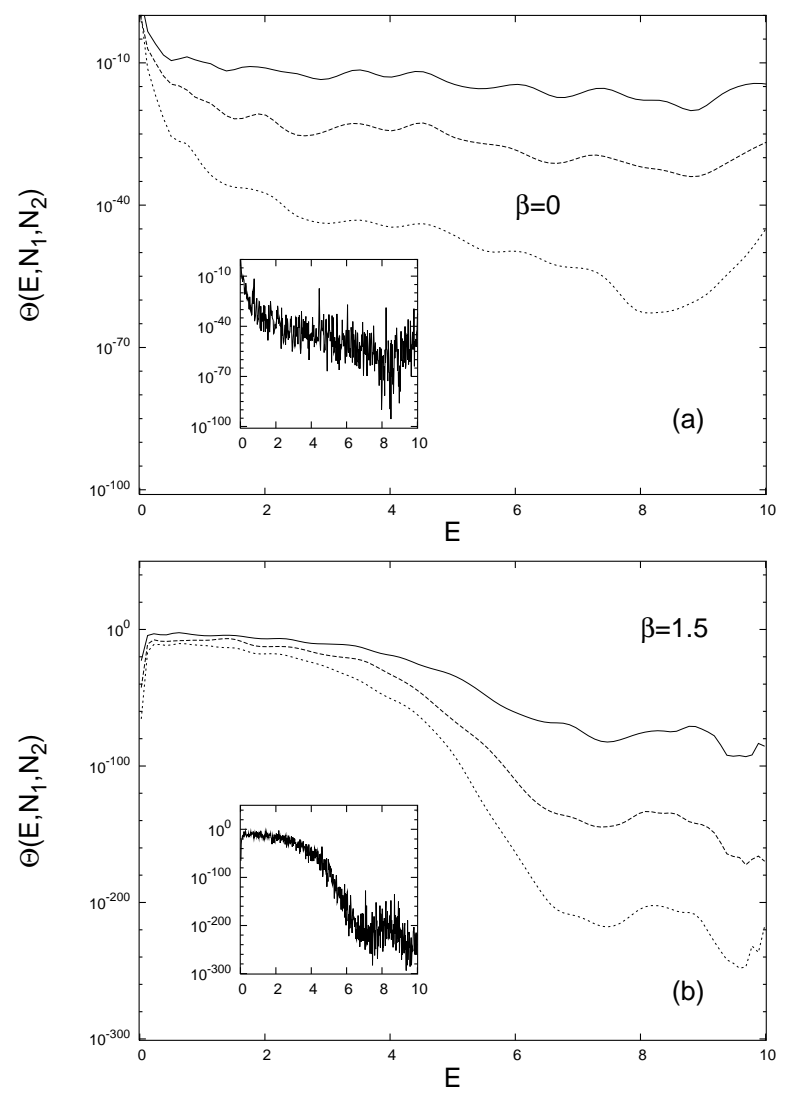

FIG. 2: Scaling function $\Theta\left(E, N_{1}, N_{2}\right)$ as function of energy $E$. Here $N_{2}=1.0 \cdot 10^{4}$ in all figures. (a) From top to bottom $N_{1}=1.5 \cdot 10^{4}, 2.0 \cdot 10^{4}, 3.0 \cdot 10^{4}$. We find that $\Theta \rightarrow 1$, i.e. $\rho_{F}(E)=0$ only for $E=0$, otherwise $\Theta \rightarrow 0$ in the thermodynamical limit. The inset shows the original data without interpolations for $N_{1}=3.0 \cdot 10^{4}$. Average performed over 120 chains. $(b)$ From top to bottom $N_{1}=2.0 \cdot 10^{4}, 3.0 \cdot 10^{4}, 4.0 \cdot 10^{4}$. In the thermodynamical limit $\Theta \rightarrow 0$, except in the region $0<E<E_{c}$ $\left(E_{c} \approx 4\right)$ where $\rho_{F}(E) \rightarrow 0$ and $\Theta \rightarrow 1$. Inset: $N_{1}=4.0 \cdot 10^{4}$. Average performed over 100 chains.

Now we proceed by numerically integrating $\rho_{F}(E)$ in order to obtain the limiting behavior of $\widetilde{\Gamma}(z)$ as $z \rightarrow 0$, using $E_{C}=4$. In Fig. 3. we show $\widetilde{\Gamma}(z)$ as a function of $z$ for $\beta=1.5$. Observe the verification of the conjecture: $\nu \approx 1.0$, implying $\alpha \approx 2.0$. We find super-diffusive motion for $\beta<1$, and ballistic motion for $\beta>1$. Superdiffusive propagation of a wave packet was also obtained in the one-dimensional Fibonacci and Thue-Morse lattices, which present deterministic aperiodic order. Since the numerical method has greater errors for smaller values of $E$, we find it difficult to obtain the density of states for small $E$ (large times). This sort of error is expected to have an influence on small values of $z$ in the Laplace transform. Another numerical problem is due to the finite size of the chain, which has important implications for $\beta>1$, as seen by the presence of the plateau in Fig. [1

Finally, Fig. 团 shows $\alpha$ as a function of $\beta$. We select $0<\beta<2$, covering both regimes. The numerical simulations are quite compatible with the stepwise behavior. The maximum deviation observed is at $\beta=0.8$, with an error of about $8 \%$, which results in $\alpha=1.39$.

\section{DISCUSSIONS AND CONCLUSIONS}

The main results reported in this work are the following: first, for the Heisenberg system we have calculated the superdiffusive and ballistic exponents with reasonable precision. Second, we have been able to associate a memory function to a Hamiltonian, through which we unite two powerful formalisms: a stochastic description and the quantum equations of motion. This result may have implications far beyond the specific example outlined in this work. Evidently, we need more simulations and experiments connecting the exponents $\alpha$ and $\nu$ in several diffusive processes in order to have a more complete picture of the validity of conjecture (8). It is important to notice that there are many phenomena associated with those described here. For example, in the ballistic regime $(\alpha=2)$, there remains 


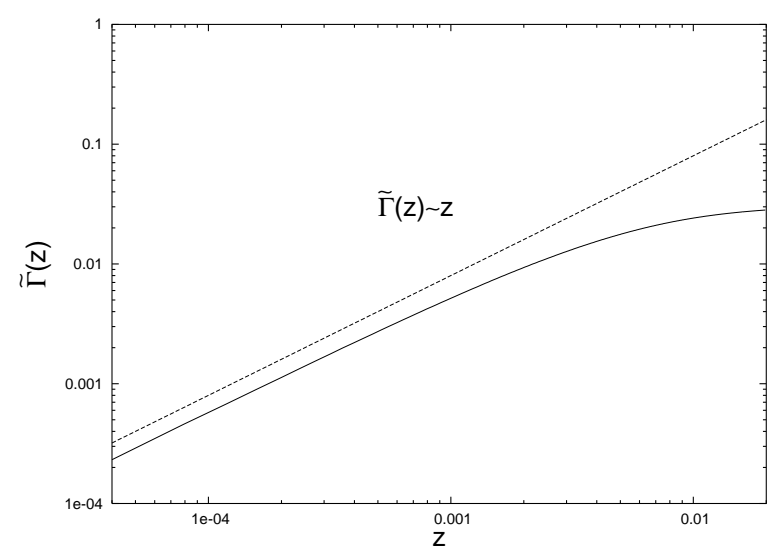

FIG. 3: The Laplace transform of the memory function $\widetilde{\Gamma}(z)$ as function of $z$. The upper curve is a guide for the eye. The lower curve is our result for $\beta=1.5$, averaged over 900 chains. In the limit $z \rightarrow 0$ we approach the expected linear behavior.

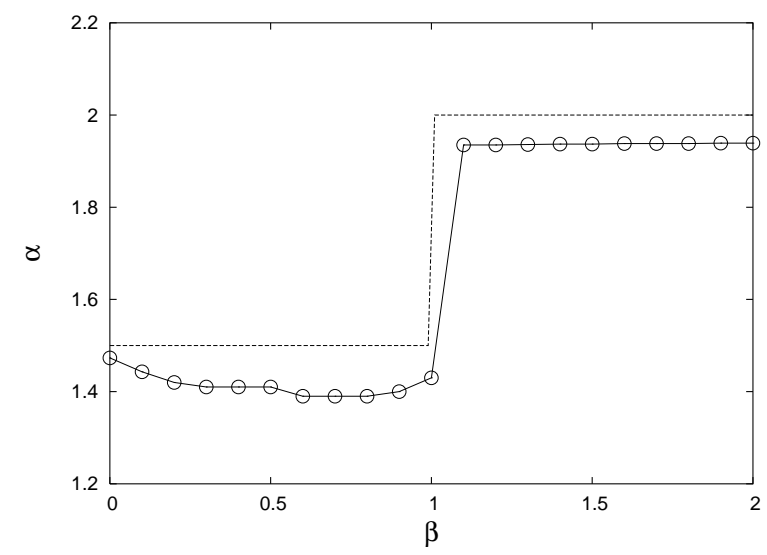

FIG. 4: Numerical data for the diffusion exponent $\alpha$ as function of $\beta$ averaged over 900 chains. The step is the expected result [15].

some open issues concerning the use of the fluctuation-dissipation theorem [22]. Since the early experiment of Kauzmann 24], situations have been found where the systems do not thermalize, usually associated with their slow dynamics 22, 24, 25, 26, 27, 28]. This slow dynamics is due to the softening of the lower fluctuating modes, here identified by the presence of a finite range of energy with extended states: $\rho_{F}(E)=0$ for $0<E<E_{c}$. This effect has been used recently [29] to obtain ballistic diffusion in ratchet devices. Another related phenomenon is chaos synchronization [30], which presents a reduction in the allowed phase space. A large time to achieve equilibrium, or the absence of some regions of the phase space, leads to the same effect. Some of its important consequences, such as violation of ergodicity [22, 31] and of the fluctuation-dissipation theorem [22], have been observed.

\section{Acknowledgments}

We thank M.L. Lyra for useful discussions. Work supported by CAPES, CNPq, FINEP, FAPDF, FACEP and FAPEAL (Brazilian Federal and States research agencies).

[1] R. Morgado, F. A. Oliveira, G. G. Batrouni and A. Hansen, Phys. Rev. Lett. 89, 100601 (2002).

[2] J. C. Flores, J. Phys.: Condens. Matter 1, 8471 (1989). 
[3] D. H. Dunlap, H. L. Wu, and P. W. Phillips, Phys. Rev. Lett. 65, 88 (1990); H.-L. Wu and P. Phillips, Phys. Rev. Lett. 66, 1366 (1991); P. W. Phillips and H.-L. Wu, Science 252, 1805 (1991).

[4] S. Gangopadhyay, A. K. Sen, J. Phys Condens. Matter 43725 (1992).

[5] P. K. Datta, D. Giri, K. Kundu, Phys. Rev. B 47, 10727 (1993).

[6] A. Schez, F. Domguez-Adame, J. Phys A:Math. Gen. 273725 (1994); A. Sánchez, E. Maciá, F. Domínguez-Adame, Phys. Rev. B 49147 (1994).

[7] E.Diez, A. Sànchez, F. Domínguez-Adame Phys. Rev. B 5014359 (1994); F. Domínguez-Adame, E.Diez, A. Sànchez, Phys. Rev. B 518115 (1995).

[8] M. Hilke, J. Phys. A: Math. Gen. 274773 (1994); M. Hilke, J. C. Flores, Phys. Rev. B 5510625 (1997).

[9] P. Ojeda, R. Huerta-Quintanilla, and M. Rodriguez-Achach, Phys. Rev. B. 65, 233102 (2002).

[10] F. A. B. F. de Moura and M. L. Lyra, Phys. Rev. Lett. 81, 3735 (1998); F. A. B. F. de Moura and M. L. Lyra, Physica A 266, 465 (1999).

[11] F. M. Izrailev and A. A. Krokhin, Phys. Rev. Lett. 82, 4062 (1999); F. M. Izrailev, A. A. Krokhin, and S. E. Ulloa, Phys. Rev. B 63, 41102 (2001).

[12] S. N. Evangelou and D. E. Katsanos, Phys. Lett. A 164,456 (1992). See also, S. N. Evangelou, A. Z. Wang, and S. J. Xiong, J. Phys.: Condens. Matter 6, 4937 (1994).

[13] V. Bellani, E. Diez, R Hey, L. Toni, , L. Tarricone, G. B. Parravicini, F. Dominguez-Adame and R. Goméz-Alcala, Phys. Rev. Lett 82, 2159 (1999); V. Bellani, E. Diez, A. Parisini, L. Tarricone, R. Hey , G .B. Parravicini , F. Dominguez-Adame, Physica E, 7 (3-4), 823 (2000).

[14] U. Kuhl, F. M. Izrailev, A. Krokhin, and H. J. Stöckmann, Appl. Phys. Lett. 77, 633 (2000).

[15] F. A. B. F. de Moura, M. D. Coutinho-Filho, E. P. Raposo, and M. L. Lyra, Phys. Rev. B 66, 014418 (2002).

[16] F. A. B. F. de Moura, M. D. Coutinho-Filho, E. P. Raposo, and M. L. Lyra, Phys. Rev. B 68, 012202 (2003).

[17] R. Kubo, Rep. Prog. Phys. 29, 255 (1966).

[18] R. Kubo, M. Toda, N. Hashitsume, Statistical Physics II, Springer (1991).

[19] H. Mori, Prog. Theor. Phys. 33, 423 (1965).

[20] M. H. Lee, J. Math. Phys. 24, 2512 (1983) - Phys. Rev. Lett. 85, 2422 (2000).

[21] P. Dean and J. L. M. Martin Proc. Roy. Soc. A259, 409 (1960); P. Dean, Rev. Mod. Phys. 44, 127 (1972).

[22] I. V. L Costa, R. Morgado, M. V. T. Lima and F. A. Oliveira, Europhysics Lett. 63, 173 (2003).

[23] P. E. de Brito, C. A. A. da Silva and H. N. Nazareno, Phys. Rev. B 51, 6096 (1995)

[24] W. Kauzmann, Chem. Rev. 43, 219 (1948).

[25] T. S. Grigera, N. E. Israeloff, Phys. Rev. Lett. 83, 5038 (1999).

[26] G. Parisi, Phys. Rev. Lett., 79, 3660 (1997).

[27] I. Santamaria-Holek, D. Reguera and J. M. Rubi, Phys. Rev. E, 63, 051106 (2001); A. Pez-Madrid, D. Reguera and J. M. Rubi, cond/mat 0210089.

[28] F. Ricci-Tersenghi, D. A. Stariolo and J. J. Arenzon, Phys. Rev. Lett.84, 4473 (2000); A. Cavagna, I. Giardine and T. Grigera, Europhys. Lett. 61, 74 (2003).

[29] J. D. Bao and Y. Z. Zhou, Phys. Rev. Lett., 91, 138104 (2003): J. D. Bao, J. Stat. Phys. 114, 503 (2004).

[30] M. Ciesla, S. P. Dias, L. Longa and F. A. Oliveira, Phys. Rev. E. 63, 065202 (2001); L. Longa, E. M. F. Curado and F.A. Oliveira, Phys. Rev. E. 54, 2201 (1996).

[31] M. H. Lee, Phys. Rev. Lett. 87250601 (2001). 